\title{
Pulmonary Artery Catheterization was not Associated with Survival Benefits in Critically III Patients with Cardiac Disease: An Analysis of the MIMIC-IV Database
}

Jie Wu

Southern Medical University Nanfang Hospital

Shiyu Zhou

Southern Medical University

Hongbin $\mathrm{Hu}$

Southern Medical University Nanfang Hospital

Yuan Zhang

Southern Medical University Nanfang Hospital

\section{Sheng An}

Southern Medical University Nanfang Hospital

\section{Tong Sha}

Southern Medical University Nanfang Hospital

Lulan Li

Southern Medical University Nanfang Hospital

Yaoyuan Zhang

Southern Medical University Nanfang Hospital

\section{Qiaobing Huang}

Southern Medical University

\section{Zhongqing Chen}

Southern Medical University Nanfang Hospital

\section{Shengli An}

Southern Medical University

Zhenhua Zeng ( $\nabla$ zhenhuazeng.2008@163.com )

Southern Medical University Nanfang Hospital https://orcid.org/0000-0002-9509-1536

\section{Research}

Keywords: pulmonary artery catheter, cardiac diseases, surgery, 28-day mortality

Posted Date: August 13th, 2021

DOl: https://doi.org/10.21203/rs.3.rs-786733/v1

License: @ (i) This work is licensed under a Creative Commons Attribution 4.0 International License. Read Full License 


\section{Abstract}

Background: It is not clear whether pulmonary artery catheter (PAC) placement is beneficial for critically ill patients with heart disease. This study aims to investigate the association of PAC use with 28-day mortality in that population.

Methods: The MIMIC-IV database was employed to identify critically ill patients with cardiac disease with or without PAC insertion. The primary outcome was 28-day mortality. Multivariate regression was modeled to examine the association between PAC and outcomes. Additionally, we examined the effect modification by cardiac surgeries. Propensity score matching (PSM) was conducted to validate our findings.

Results: No improvement in 28-day mortality was observed among the PAC group compared to the non-PAC group (odds ratio=1.18, 95\% $\mathrm{Cl}=1.00-1.38, P=0.049)$. When stratified by cardiac surgeries, the results were consistent. Patients in the PAC group had fewer ventilation-free days and vasopressor-free days than those in the non-PAC group after surgery stratification. In surgical patients, PAC insertion was not associated with the occurrence of acute kidney injury (AKI), and was associated with a higher daily fluid input (mean difference $=0.13,95 \% \mathrm{Cl}=0.05-0.20, P=0.001$ ). In non-surgical patients, the PAC group had a higher risk of $\mathrm{AKI}$ occurrence (odds ratio=1.94, $95 \% \mathrm{Cl}=1.32-2.84, P=0.001$ ).

Conclusion: PAC placement was not associated with survival benefits in critically ill patients with cardiac diseases, either in surgical and non-surgical patients.

\section{Background}

Pulmonary artery catheter (PAC) has been utilized in the management of critically ill and high-risk surgical patients since $\mathrm{Dr} \mathrm{HJ}$ Swan and Dr William Ganz first introduced the flow-directed balloon-tipped catheter in 1970[1]. A PAC provides the intensivist with continuous monitoring of critical hemodynamic data that includes cardiac output (CO), mixed venous oxygen saturation, and intrapulmonary and intracardiac pressures[1]. PAC use was considered to be useful for guiding the administration of fluids, inotropes, and diuretics. Therefore, it was thought to improve potential clinical outcomes.

However, PAC insertion has been associated with numerous complications, including air embolism, dysrhythmias, and pulmonary artery rupture[2]. Notably, the information provided by PAC might be inaccurate, and the misinterpretation of data influences interventions and outcomes. Over the past decades, the use of PAC has varied markedly between different clinical settings. The results from the ESCAPE trial showed that PAC did not affect overall mortality and hospitalization in patients with congestive heart failure[3]. The GUSTO trial found that PAC was associated with increased mortality in patients with acute coronary syndromes[4]. Systematic review and meta-analyses from randomized controlled trials (RCTs) have failed to show a clear benefit of PAC for in-hospital morbidity and mortality [2, 5]. In contrast, Randall et al. first demonstrated a benefit of PAC use in trauma patients[6]. Similarly, a retrospective cohort study showed that the use of PAC during adult cardiac surgery was associated with a decreased length of stay and reduced cardiopulmonary morbidity, which suggests an overall potential benefit of PAC placement[7]. More recently, a prospective cohort study containing 11820 patients showed that PAC use was not associated with improved operative mortality in patients undergoing open cardiac surgeries[8].

Until now, data describing the outcomes of PAC use in critically ill patients with cardiac diseases have been limited and conflicting. Thus, the current study was carried out to investigate the impact of PAC placement on the outcomes of critically ill adult patients with cardiac diseases.

\section{Methods}

\section{Study design and population}

This study was a retrospective, observational study from the Multiparameter Intelligent Monitoring in Intensive Care IV (MIMICIV) database (version 0.4) from 2008 to 2019[9]. One author who finished the test in PHYSIONET (CITI Certification number 35931520) obtained access to the database and was responsible for data extraction. There were more than sixty thousand 
patients with ICU admission in the database. Adult patients with cardiac diseases, including heart valve disease, coronary artery disease, myocardial infarction, heart failure, cardiogenic shock, and arrhythmia, were screened in the analysis. For patients who were admitted to the ICU more than once, we included only the first ICU admission. We excluded patients who spent less than 24 $\mathrm{h}$ in the ICU. Included patients for whom initial PAC insertion was completed within $24 \mathrm{~h}$ after ICU admission were classified as the PAC group, with the rest defined as the non-PAC group. In our study, we extracted patient parameters including age, gender, ethnicity, admission type, insurance condition, marital status, weight, and severity at admission as measured by the first 24-h Simplified Acute Physiology Score II (SAPS) score, Sequential Organ Failure Assessment (SOFA) score, and Charlson Comorbidity Index (CCl) score. Vital signs included heart rate, respiratory rate, temperature, SpO2, and mean arterial blood pressure (MAP). Laboratory variables, including lactate, white blood cell (WBC) counts, platelet counts, and creatinine, were measured during the first $24 \mathrm{~h}$ in the ICU. Information on cardiac surgeries and the use of mechanical ventilation and vasopressors during the first $24 \mathrm{~h}$ was also recorded. Comorbidities including renal disease, chronic obstructive pulmonary disease (COPD), and cancer were also collected for analysis based on the recorded ICD-10 codes in the MIMIC-IV database.

\section{Primary Outcome And Secondary Outcomes}

The primary outcome in the present study was 28-day mortality. Secondary outcomes included 90-day mortality, the incidence of AKI, the number of ventilator-free and vasopressor-free days within 28 days after ICU admission, and daily fluid input.

\section{Statistical analysis}

Baseline characteristics and clinical parameters were summarized between the PAC and non-PAC groups. Continuous variables were described as the mean (standard variance) or median (interquartile range) depending on whether they were normally distributied or skewed. Categorical variables were described as counts (\%). Differences in variables between the PAC group and the non-PAC group were compared using the Student's t-test, chi-square test, and Wilcoxon rank sum tests as appropriate. Multivariate logistic regression was modeled to examine the association between PAC and outcomes. To interpret the findings of the models, we estimated the odds ratios (ORs) and corresponding $95 \%$ confidence intervals (Cls) with confounding factor adjustments, including age, gender, ethnicity, admission type, insurance, marital status, weight, SAPS-II score, SOFA score, CCI score, heart rate, respiratory rate, temperature, $\mathrm{SpO}_{2}$, MAP, lactate, WBC counts, platelet counts, creatinine, cardiac surgeries, the use of mechanical ventilation and vasopressors and comorbidities.

We conducted propensity score matching (PSM) to reduce potential bias. Propensity scores, the likelihood of each individual receiving PAC, were calculated using logistic regression in which the exposure (PAC) was treated as the outcome in the model and regressed with the covariates mentioned above. After PSM, the matching populations between the PAC and non-PAC groups trended to balance, and the balance properties were evaluated by standard mean difference (SMD). When the SMD for each variable incorporated in the PSM model was less than 0.1 , the characteristics among the two groups were considered balanced[10].

As in previous reports, we focused the group comparisons on disparities between populations with or without cardiac surgery because these conditions provide interpretation of PAC disparities. Thus, for primary and secondary outcomes, differences in groups were compared between cardiac surgery and non-cardiac surgery groups. To further assess the effect modification by cardiac surgery, we stratified the multivariate logistic and PSM analyses according to their status of surgery.

A sensitivity analysis was conducted to evaluate whether the initial timing of PAC impacted our results. We categorized patients whose initial timing of PAC was less than or more than three hours into the PAC group and compared them against the non-PAC group. Moreover, we further examined the association between different durations ( $\leq 1$ and $>1$ day) of PAC and outcomes. Missing values were imputed by multiple imputation and complete case analyses were also performed as sensitivity analyses.

All analyses were performed by R (version 4.0.5) and SAS (version 9.4). A two-sided $P$ value less than 0.05 was an indicator of statistical significance. 


\section{Results}

\section{Baseline characteristics}

After reviewing the data of 69619 MIMIC-IV adult admissions, a total of 19158 patients with cardiac diseases were identified. Finally, we included 11887 patients in our study cohort (Fig. 1). Among the study cohort, 2727 patients (22.9\%) received PAC within $24 \mathrm{~h}$ after ICU admission. The duration of use of PAC was $21.6 \mathrm{~h}$ (IQR, 16.8-40.8). The time to initial PAC insertion was $4.8 \mathrm{~h}(\mathrm{IQR}=2.4-7.2)$. The baseline characteristics of the cohort are summarized in Table 1. Patients in the PAC group had significantly higher SAPS-II scores (33.0 vs. 32.0, $P=0.002)$ and higher SOFA scores (6.0 vs. 4.0, $P=0.027$ ) but lower CCI scores (5.0 vs. $6.0, P=0.007$ ) on admission. A larger percentage of the PAC patients received mechanical ventilation ( $88.9 \%$ vs. $33.1 \%$, $P<0.001)$ and vasopressor treatment $(85.9 \%$ vs. $34.1 \%, P<0.001)$ during the first $24 \mathrm{~h}$ of their ICU stay. The two cohorts were balanced for all variables after matching (Table S1). 
Table 1

Baseline demographics of the original cohort

\begin{tabular}{|c|c|c|c|c|}
\hline Characteristics & Non-PAC & PAC & Total & $P$ \\
\hline Age(yr) median (IQR) & $74.6(65.1-83.5)$ & $71.5(62.9-79.4)$ & $73.8(64.4-82.5)$ & $<0.001$ \\
\hline Male (\%) & $5478(59.8)$ & $1845(67.7)$ & 7323(61.6) & $<0.001$ \\
\hline White (\%) & $6358(69.4)$ & $2001(73.4)$ & $8359(70.3)$ & $<0.001$ \\
\hline Emergency (\%) & $5555(60.6)$ & $595(21.8)$ & $6150(51.7)$ & $<0.001$ \\
\hline Insurance (\%) & $5521(60.3)$ & $1420(52.1)$ & $6941(58.4)$ & $<0.001$ \\
\hline Married (\%) & $4485(49.0)$ & $1619(59.4)$ & 6104(51.4) & $<0.001$ \\
\hline Weight (kg) median (IQR) & $79.9(67.0-94.9)$ & $86.0(73.5-99.3)$ & $81.1(68.5-95.9)$ & $<0.001$ \\
\hline SAPS-II, median (IQR) & $32.0(26.0-39.0)$ & $33.0(27.0-41.0)$ & $32.0(26.0-40.0)$ & $<0.001$ \\
\hline SOFA, median (IQR) & $4.0(3.0-6.0)$ & $6.0(5.0-8.0)$ & $5.0(3.0-6.0)$ & $<0.001$ \\
\hline CCl, median (IQR) & $6.0(5.0-8.0)$ & $5.0(4.0-7.0)$ & $6.0(5.0-8.0)$ & $<0.001$ \\
\hline Heart rate (bpm) median (IQR) & $82.0(72.2-93.6)$ & $82.2(77.0-88.8)$ & $82.1(73.5-92.2)$ & 0.480 \\
\hline Respiratory rate (/min) median (IQR) & $18.9(16.8-21.5)$ & $17.4(15.8-19.2)$ & $18.5(16.5-21.0)$ & $<0.001$ \\
\hline Temperature $\left({ }^{\circ} \mathrm{C}\right)$ median $(\mathrm{IQR})$ & $36.7(36.5-37.0)$ & $36.7(36.5-37.0)$ & $36.7(36.5-37.0)$ & 0.193 \\
\hline $\mathrm{SpO}_{2}(\%)$ median (IQR) & $97.0(95.6-98.3)$ & $97.9(96.9-98.8)$ & $97.3(95.9-98.4)$ & $<0.001$ \\
\hline MAP (mmHg), median (IQR) & $78.5(72.8-85.8)$ & $74.6(71.2-78.3)$ & $77.2(72.3-83.8)$ & $<0.001$ \\
\hline Lactate (mmol/L), median (IQR) & $1.7(1.3-2.4)$ & $2.0(1.6-2.6)$ & $1.8(1.4-2.5)$ & $<0.001$ \\
\hline White blood cell $(\mathrm{WBC})\left(\times 10^{9}\right)$, median (IQR) & $10.7(8.0-14.3)$ & $12.3(9.7-15.5)$ & $11.1(8.4-14.6)$ & $<0.001$ \\
\hline Platelets $\left(\times 10^{12}\right)$, median (IQR) & 193.8(146.3-253.3) & $150.8(122.0-190.0)$ & $182.0(138.0-239.0)$ & $<0.001$ \\
\hline Creatinine (mg/dl), median (IQR) & $1.1(0.8-1.6)$ & $1.0(0.8-1.3)$ & $1.0(0.8-1.5)$ & $<0.001$ \\
\hline Cardiac surgery (\%) & $1358(14.8)$ & $2347(86.1)$ & $3705(31.2)$ & $<0.001$ \\
\hline Mv use (1st $24 \mathrm{~h})$ & $3034(33.1)$ & 2424(88.9) & $5458(45.9)$ & $<0.001$ \\
\hline Vasopressor use (1st 24 h) & $3127(34.1)$ & 2342(85.9) & $5469(46.0)$ & $<0.001$ \\
\hline \multicolumn{5}{|l|}{ Comorbidities (\%) } \\
\hline Renal disease & $2538(27.7)$ & $562(20.6)$ & $3100(26.1)$ & $<0.001$ \\
\hline Cancer & $1179(12.9)$ & 104(3.8) & $1283(10.8)$ & $<0.001$ \\
\hline COPD & 2805(30.6) & $796(29.2)$ & $3601(30.3)$ & 0.153 \\
\hline
\end{tabular}

\section{Primary Outcome}

The 28-day mortality rates for the PAC and non-PAC groups were $5.0 \%$ and $12.7 \%$, respectively (Table 2 ). The association between PAC and outcomes was shown in Fig. 2. Before PSM, PAC was significantly associated with an increased risk of 28-day mortality $(\mathrm{OR}=1.18,95 \% \mathrm{Cl}=1.00-1.38, P=0.049)$, but this became insignificant after $\mathrm{PSM}(\mathrm{OR}=0.82,95 \% \mathrm{Cl} 0.63-1.08, P=$ 0.155). We also examined the effect modification of cardiac surgeries on the association between PAC and 28-day mortality 
(Table 3). In patients with cardiac surgery, PAC insertion status was not associated with 28-day mortality before and after PSM $(P>0.678)$. In patients who underwent cardiac surgery, the mortality rates for the PAC and non-PAC groups were $24.5 \%$ vs. $14.7 \%$, respectively. A significantly increased risk of 28-day mortality was observed in patients with $\mathrm{PAC}$ insertion $(\mathrm{OR}=1.26,95 \% \mathrm{Cl}=$ $1.05-1.51, P=0.015)$ but not after PSM (OR $=1.29,95 \% \mathrm{Cl}=0.90-1.84, P=0.171)$. These data indicated that PAC insertion was not associated with an improvement in 28-day mortality in patients with cardiac diseases regardless of whether they underwent surgery.

Table 2

Primary and secondary outcomes

\begin{tabular}{|c|c|c|c|c|c|c|c|c|c|}
\hline \multirow[t]{2}{*}{ Outcomes } & \multicolumn{3}{|l|}{ Total } & \multicolumn{3}{|l|}{ Non-surgery } & \multicolumn{3}{|l|}{ Surgery } \\
\hline & Non-PAC & PAC & $P$ & Non-PAC & PAC & $P$ & Non-PAC & PAC & $P$ \\
\hline $\begin{array}{l}\text { 28-day } \\
\text { mortality (\%) }\end{array}$ & 1166(12.7) & 135(5.0) & $\begin{array}{l}< \\
0.001\end{array}$ & 1148(14.7) & $93(24.5)$ & $\begin{array}{l}< \\
0.001\end{array}$ & $18(1.3)$ & $42(1.8)$ & 0.281 \\
\hline $\begin{array}{l}\text { 90-day } \\
\text { mortality (\%) }\end{array}$ & $1224(13.4)$ & $146(5.4)$ & $\begin{array}{l}< \\
0.001\end{array}$ & 1206(15.5) & $95(25.0)$ & $\begin{array}{l}<.001 \\
0.001\end{array}$ & 18(1.3) & $51(2.2)$ & 0.066 \\
\hline $\begin{array}{l}\text { Incidence of } \\
\text { AKI (\%) }\end{array}$ & $6319(69.0)$ & $2204(80.8)$ & $\begin{array}{l}<.001 \\
0.001\end{array}$ & $5353(68.6)$ & $326(85.8)$ & $\begin{array}{l}< \\
0.001\end{array}$ & $966(71.1)$ & $1878(80.0)$ & $\begin{array}{l}< \\
0.001\end{array}$ \\
\hline $\begin{array}{l}\text { Ventilation- } \\
\text { free days } \\
\text { (sd) }\end{array}$ & $24.8(5.1)$ & $26.0(4.4)$ & $\begin{array}{l}< \\
0.001\end{array}$ & $24.2(5.4)$ & 23.3(5.8) & 0.017 & $27.2(2.7)$ & $26.3(4.1)$ & $\begin{array}{l}<.001 \\
0.001\end{array}$ \\
\hline $\begin{array}{l}\text { Vasopressor- } \\
\text { free days } \\
\text { (sd) }\end{array}$ & $25.7(4.1)$ & $25.9(4.0)$ & 0.007 & $25.0(4.5)$ & $23.9(5)$ & $\begin{array}{l}< \\
0.001\end{array}$ & $27.2(2.2)$ & $26.2(3.7)$ & $\begin{array}{l}<.001 \\
0.001\end{array}$ \\
\hline $\begin{array}{l}\text { Daily fluid } \\
\text { input (sd) }\end{array}$ & $1.6(1.2)$ & 1.6(1.3) & 0.073 & $1.7(1.3)$ & $2.3(1.8)$ & $\begin{array}{l}< \\
0.001\end{array}$ & $1.3(0.8)$ & $1.5(1.2)$ & $\begin{array}{l}<.001 \\
0.00\end{array}$ \\
\hline
\end{tabular}


Table 3

The effect modification of cardiac surgery on the association of PAC and outcomes

\begin{tabular}{|c|c|c|c|c|c|c|c|c|}
\hline \multirow[t]{2}{*}{ Outcomes } & \multicolumn{4}{|c|}{ Before PSM } & \multicolumn{4}{|l|}{ After PSM } \\
\hline & Estimate & LOW & UP & $P$ & Estimate & LOW & UP & $P$ \\
\hline \multicolumn{9}{|l|}{ Cardiac surgery } \\
\hline \multicolumn{9}{|l|}{ Odd ratios } \\
\hline 28-day mortality & 1.10 & 0.69 & 1.76 & 0.678 & 1.00 & 0.47 & 2.11 & 1.000 \\
\hline 90-day mortality & 1.22 & 0.79 & 1.89 & 0.367 & 1.14 & 0.56 & 2.36 & 0.713 \\
\hline Incidence of AKI & 0.94 & 0.84 & 1.04 & 0.229 & 0.99 & 0.83 & 1.17 & 0.862 \\
\hline \multicolumn{9}{|l|}{ Mean difference } \\
\hline Ventilation-free days & -0.35 & -0.55 & -0.14 & 0.001 & -0.38 & -0.59 & -0.18 & $<0.001$ \\
\hline Vasopressor-free days & -0.38 & -0.59 & -0.17 & 0.000 & -0.48 & -0.70 & -0.27 & $<0.001$ \\
\hline Daily fluid input & 0.10 & 0.02 & 0.18 & 0.011 & 0.13 & 0.05 & 0.20 & 0.001 \\
\hline \multicolumn{9}{|l|}{ Non-cardiac surgery } \\
\hline \multicolumn{9}{|l|}{ Odd ratios } \\
\hline 28-day mortality & 1.26 & 1.05 & 1.51 & 0.015 & 1.29 & 0.90 & 1.84 & 0.171 \\
\hline 90-day mortality & 1.20 & 1.00 & 1.44 & 0.053 & 1.24 & 0.87 & 1.76 & 0.242 \\
\hline Incidence of AKI & 1.25 & 1.05 & 1.50 & 0.014 & 1.94 & 1.32 & 2.84 & 0.001 \\
\hline \multicolumn{9}{|l|}{ Mean difference } \\
\hline Ventilation-free days & -0.69 & -1.20 & -0.17 & 0.009 & -0.58 & -1.23 & 0.07 & 0.081 \\
\hline Vasopressor-free days & -1.05 & -1.48 & -0.61 & 0.000 & -0.92 & -1.51 & -0.33 & 0.002 \\
\hline Daily fluid input & 0.02 & -0.15 & 0.18 & 0.860 & 0.10 & -0.15 & 0.35 & 0.432 \\
\hline
\end{tabular}

\section{Secondary Outcomes}

PAC insertion was associated with an increased 90-day mortality. However, this significance was not maintained after PSM (Fig. 2). Patients in the PAC group had fewer ventilation-free days (23.3 vs. $24.2 \%, P=0.017$ in the non-surgery group and 26.3 vs. $27.2 \%, P<0.001$ in the surgery group) and vasopressor-free days ( 23.9 vs. $25.0 \%, P<0.001$ in non-surgery group and 26.2 vs. $27.2 \%, P<0.001$ in the surgery group) than those in the non-PAC group after surgery stratification (Table 2). When stratified by cardiac surgeries (Table 3), in surgical patients, PAC insertion was not associated with the occurrence of AKI and was associated with a higher daily fluid input before (mean difference $=0.10,95 \% \mathrm{Cl}=0.02-0.18, P=0.011$ ) and after PSM (mean difference = $0.13,95 \% \mathrm{Cl}=0.05-0.20, P=0.001)$. In non-surgical patients, PAC group had a higher risk of AKI occurrence before $(\mathrm{OR}=1.25$, $95 \% \mathrm{Cl}=1.05-1.50, P=0.014)$ and after PSM $(\mathrm{OR}=1.94,95 \% \mathrm{Cl}=1.32-2.84, P=0.001)$.

\section{Sensitivity Analyses And Additional Analyses}

Sensitivity analysis showed similar results regarding the initial timing of PAC insertion (Table S2). Additional analysis showed that patients with a PAC duration of more than 1 day had a higher mortality rate and a longer ventilation time (Table S3). These results were maintained in the complete case analysis (Table S4). 


\section{Discussion}

The major findings of this study are that PAC placement was not associated with a survival benefit in critically ill patients with cardiac diseases, either in surgical or non-surgical patients. Furthermore, those who received PAC insertion also had fewer vasopressor- and ventilation-free days than the non-PAC group. In surgical patients, PAC insertion had no effect on AKI occurrence but was associated with a higher volume of fluid administration. In non-surgical patients, PAC insertion was associated with a higher risk of AKI occurrence, while no association with fluid input was observed.

Since its introduction, PAC has been used in the management of critically ill patients. Importantly, PAC measures, particularly in critically ill patients, are frequently inaccurate[11]. Misinterpretation of data is generally accepted to be common and can potentially lead to mismanagement of the patients. Thus, PAC-directed therapy, including the use of fluids and inotropes, may be ineffective or harmful in some statuses[12].

Though Shaw and colleagues showed that PAC use was associated with an overall potential benefit during adult cardiac surgery [7], the majority of studies have shown no mortality benefit or higher mortality when assessing the influence of PAC placement on critically ill patients with cardiac surgeries[12-15]. Despite the debate about PAC use in the management of cardiac surgery, an international survey showed that practitioners preferred using PAC for most cardiac surgeries[16]. In the present study, no significant difference was observed between the PAC group and the non-PAC group in patients undergoing cardiac surgeries. Similarly, no survival benefit has been definitely confirmed in non-surgical patients with cardiac diseases. Previously, the ESCAPE trial found that PAC use had no effects on overall mortality and hospitalization in patients with congestive heart failure[3]. Gabriel et al. conducted a retrospective cohort study and found that PAC use was associated with higher mortality in patients with heart failure[17]. In those with cardiogenic shock, PAC was associated with lower mortality and in-hospital cardiac arrest[17]. Recently, another retrospective study evaluating PAC use in patients with acute myocardial infarction-cardiogenic shock excluding cardiac surgery showed that the PAC cohort had a higher in-hospital mortality, longer length of stay, higher hospitalization costs, and fewer discharges to home[18]. Here, in this study, no mortality benefit of PAC use was identified in nonsurgical patients with cardiac diseases. However, as PAC is not routinely indicated in these patients and there is currently no consensus on the use of PAC in these patients[19,20], future studies are warranted.

In this study, patients in the PAC group had higher severity of illness scores and were more likely to receive mechanical ventilation and vasopressors at admission. Although these factors indicated a more severe condition, we used PSM analysis and found no mortality benefit of PAC insertion after adjustment for confounding factors. Moreover, the initial timing of PAC insertion had little effect on clinical outcomes. Of note, a longer indwelling time of PAC was associated with a worse outcome.

PAC measurement of real-time parameters can guide timely and accurate interventions, including the use of fluids, vasopressors, and inotropes. On the other hand, it may also lead to a state of overtreatment. We found that PAC placement was associated with a longer support of ventilation and vasopressors. Differences were observed between surgical and non-surgical patients regarding the management of fluid and occurrence of AKI. Consistent with a previous cohort study[8], PAC insertion had no effect on AKI occurrence in cardiac surgery patients. While in non-surgical patients, PAC insertion was associated with a higher risk of AKI occurrence. Similar results were demonstrated in patients with heart failure[17].

When comparing mortality rates for different subgroups considering whether or not they underwent surgeries, a seemingly strange pattern can be observed: for surgery and non-surgery subgroups, mortality rates in PAC group were higher than those in the non-PAC group, but the total mortality rate was higher in the non-PAC group. This constitutes a textbook example of a statistical phenomenon known as Simpson's paradox which refers to the observation that aggregating data across subpopulations may yield opposite trends (and thus lead to reversed conclusions) when considering subpopulations separately[21]. The key to understanding the phenomenon lies in the fact that we are dealing with relative frequencies. However, such percentages conceal the absolute numbers of patients within each age group. Considering these absolute numbers sheds light on how the phenomenon can arise: the distribution of cases with PAC or without PAC differs significantly between the two subgroups. Particularly, the surgery group recorded a much higher proportion of patients with PAC than the non-surgery group. In summary, the larger share of PAC insertion among surgery patients combined with the fact that the non-surgery patients are at 
higher risk of death, explains the mismatch between total and subgroup mortality rates and thus gives rise to Simpson's paradox in the data.

Several limitations in the present study should not be neglected. First, this is an observational single-center study retrospectively performed on electronic health record data. The generalizability of these findings to other institutions requires more investigation. Second, compared with the non-PAC group, patients in the PAC group had a higher severity of illness. Though PSM was used to balance these covariates in terms of their baseline risk, there may still be unmeasured confounders that bias the study findings. Well-designed RCTs with large sample sizes are needed in the future. Third, data surrounding pulmonary artery pressures were limited in this dataset; thus, we failed to perform further analysis. Fourth, in addition to PAC utilization, less invasive $\mathrm{CO}$ measurement techniques, such as FloTrac/Vigileo and PiCCO monitoring, have been increasingly used in clinical practice. However, there were no data available in the mimic database, and thus we could not perform further analysis.

\section{Conclusions}

In critically ill patients with heart diseases, no survival benefits were observed in patients with PAC insertion, regardless of whether they underwent surgery. As suggested, there is no indication for the routine use of PAC, and thus clinicians should carefully consider the clinical risks and benefits on a patient by patient basis[22]. However, assessment of PAC effectiveness is not straightforward, given use/nonuse biases and considerable differences in the interpretation of PAC measurements among practitioners[23]. The use of PAC should not be totally abandoned, and a future topic of research should be define the most suitable patients.

\section{List Of Abbreviations}

\begin{tabular}{|ll|}
\hline PAC & Pulmonary artery catheter \\
\hline CO & Cardiac output \\
\hline RCTS & Randomized controlled trials \\
\hline MIMIC-IV & Multiparameter Intelligent Monitoring in Intensive Care IV \\
\hline SAPS & Simplified Acute Physiology Score II \\
\hline SOFA & Sequential Organ Failure Assessment \\
CCI & Charlson Comorbidity Index \\
MAP & Mean arterial blood pressure \\
WBC & White blood cell \\
\hline COPD & Chronic obstructive pulmonary disease \\
AKI & Acute kidney injury \\
\hline PSM & Propensity score matching \\
\hline SMD & Standard mean difference \\
\hline
\end{tabular}

\section{Declarations}

\section{Funding}

This study was supported by the Natural Science Foundation of Guangdong Province, China (grant nos. $2021 \mathrm{~A} 1515010002$ and 2020A151501361), the National Natural Science Foundation of China (grant nos. 82002078 and 81871604), and the Presidential Foundation of Nanfang Hospital, Southern Medical University, Guangzhou, China (grant no. 2016C026).

\section{Conflicts of interest}


The authors declare that they have no competing interests.

\section{Availability of data and material}

The datasets used in the present study are available from the first author and corresponding authors on reasonable request.

\section{Code availability}

Not applicable.

\section{Authors' contributions}

W.J. designed the study and contributed to writing this manuscript. Z.S collected and analyzed data. H.H., Z.Y and S.T helped with data analysis. A.S, L.L. and Z.Y.contributed to writing this manuscript. Q.H. and Z.C. drafted and provided critical revision of the article. A.S. and Z.Z. designed and supervised the study. All the authors provided final approval of the version submitted for publication.

\section{Ethics approval}

The MIMIC IV database used in the present study was approved by the Institutional Review Boards (IRB) of the Massachusetts Institute of Technology and does not contain protected health information.

\section{Consent to participate}

Not applicable.

\section{Consent for publication}

Not applicable.

\section{References}

1. Swan HJ, Ganz W, Forrester J, Marcus H, Diamond G, Chonette D. Catheterization of the heart in man with use of a flowdirected balloon-tipped catheter. N Engl J Med. 1970;283(9):447-51.

2. Rajaram SS, Desai NK, Kalra A, Gajera M, Cavanaugh SK, Brampton W, Young D, Harvey S, Rowan K. Pulmonary artery catheters for adult patients in intensive care. Cochrane Database Syst Rev 2013(2):CD003408.

3. Binanay C, Califf RM, Hasselblad V, O'Connor CM, Shah MR, Sopko G, Stevenson LW, Francis GS, Leier CV, Miller LW, et al. Evaluation study of congestive heart failure and pulmonary artery catheterization effectiveness: the ESCAPE trial. JAMA. 2005;294(13):1625-33.

4. Cohen MG, Kelly RV, Kong DF, Menon V, Shah M, Ferreira J, Pieper KS, Criger D, Poggio R, Ohman EM, et al. Pulmonary artery catheterization in acute coronary syndromes: insights from the GUSTO IIb and GUSTO III trials. Am J Med. 2005;118(5):482-8.

5. Shah MR, Hasselblad V, Stevenson LW, Binanay C, O'Connor CM, Sopko G, Califf RM. Impact of the pulmonary artery catheter in critically ill patients: meta-analysis of randomized clinical trials. JAMA. 2005;294(13):1664-70.

6. Friese RS, Shafi S, Gentilello LM. Pulmonary artery catheter use is associated with reduced mortality in severely injured patients: a National Trauma Data Bank analysis of 53,312 patients. Crit Care Med. 2006;34(6):1597-601.

7. Shaw AD, Mythen MG, Shook D, Hayashida DK, Zhang X, Skaar JR, lyengar SS, Munson SH. Pulmonary artery catheter use in adult patients undergoing cardiac surgery: a retrospective, cohort study. Perioper Med (Lond). 2018;7:24.

8. Brown JA, Aranda-Michel E, Kilic A, Serna-Gallegos D, Bianco V, Thoma FW, Sultan I. The impact of pulmonary artery catheter use in cardiac surgery. $J$ Thorac Cardiovasc Surg 2021.

9. Johnson A, Bulgarelli L, Pollard T, Horng S, Celi L, Mark R: MIMIC-IV (version 0.4). In.: PhysioNet; 2020. 
10. Zhang Z, Kim HJ, Lonjon G, Zhu Y. written on behalf of AMEB-DCTCG: Balance diagnostics after propensity score matching. Ann Transl Med. 2019;7(1):16.

11. Eisenberg PR, Jaffe AS, Schuster DP. Clinical evaluation compared to pulmonary artery catheterization in the hemodynamic assessment of critically ill patients. Crit Care Med. 1984;12(7):549-53.

12. Schwann NM, Hillel Z, Hoeft A, Barash P, Mohnle P, Miao Y, Mangano DT. Lack of effectiveness of the pulmonary artery catheter in cardiac surgery. Anesth Analg. 2011;113(5):994-1002.

13. Chiang Y, Hosseinian L, Rhee A, Itagaki S, Cavallaro P, Chikwe J. Questionable benefit of the pulmonary artery catheter after cardiac surgery in high-risk patients. J Cardiothorac Vasc Anesth. 2015;29(1):76-81.

14. Ramsey SD, Saint S, Sullivan SD, Dey L, Kelley K, Bowdle A. Clinical and economic effects of pulmonary artery catheterization in nonemergent coronary artery bypass graft surgery. J Cardiothorac Vasc Anesth. 2000;14(2):113-8.

15. Resano FG, Kapetanakis El, Hill PC, Haile E, Corso PJ. Clinical outcomes of low-risk patients undergoing beating-heart surgery with or without pulmonary artery catheterization. J Cardiothorac Vasc Anesth. 2006;20(3):300-6.

16. Ikuta K, Wang Y, Robinson A, Ahmad T, Krumholz HM, Desai NR. National Trends in Use and Outcomes of Pulmonary Artery Catheters Among Medicare Beneficiaries, 1999-2013. JAMA Cardiol 2017, 2(8):908-913.

17. Hernandez GA, Lemor A, Blumer V, Rueda CA, Zalawadiya S, Stevenson LW, Lindenfeld J. Trends in Utilization and Outcomes of Pulmonary Artery Catheterization in Heart Failure With and Without Cardiogenic Shock. J Card Fail. 2019;25(5):364-71.

18. Vallabhajosyula S, Shankar A, Patlolla SH, Prasad A, Bell MR, Jentzer JC, Arora S, Vallabhajosyula S, Gersh BJ, Jaffe AS, et al. Pulmonary artery catheter use in acute myocardial infarction-cardiogenic shock. ESC Heart Fail. 2020;7(3):1234-45.

19. Rossello X, Vila M, Rivas-Lasarte M, Ferrero-Gregori A, Sans-Rosello J, Duran-Cambra A, Sionis A. Impact of Pulmonary Artery Catheter Use on Short- and Long-Term Mortality in Patients with Cardiogenic Shock. Cardiology. 2017;136(1):61-9.

20. Sionis A, Rivas-Lasarte M, Mebazaa A, Tarvasmaki T, Sans-Rosello J, Tolppanen H, Varpula M, Jurkko R, Banaszewski M, Silva-Cardoso J, et al. Current Use and Impact on 30-Day Mortality of Pulmonary Artery Catheter in Cardiogenic Shock Patients: Results From the CardShock Study. J Intensive Care Med. 2020;35(12):1426-33.

21. Simpson EHJJotRSSSB. The interpretation of interaction in contingency tables. 1951, 13(2):238-241.

22. Youssef N, Whitlock RP. The Routine Use of the Pulmonary Artery Catheter Should Be Abandoned. Can J Cardiol. 2017;33(1):135-41.

23. Wakefield B, Geube M. Commentary: The uncertain fate of the pulmonary artery catheter in cardiac surgery: The difference is in the exceptions. J Thorac Cardiovasc Surg 2021.

\section{Figures}




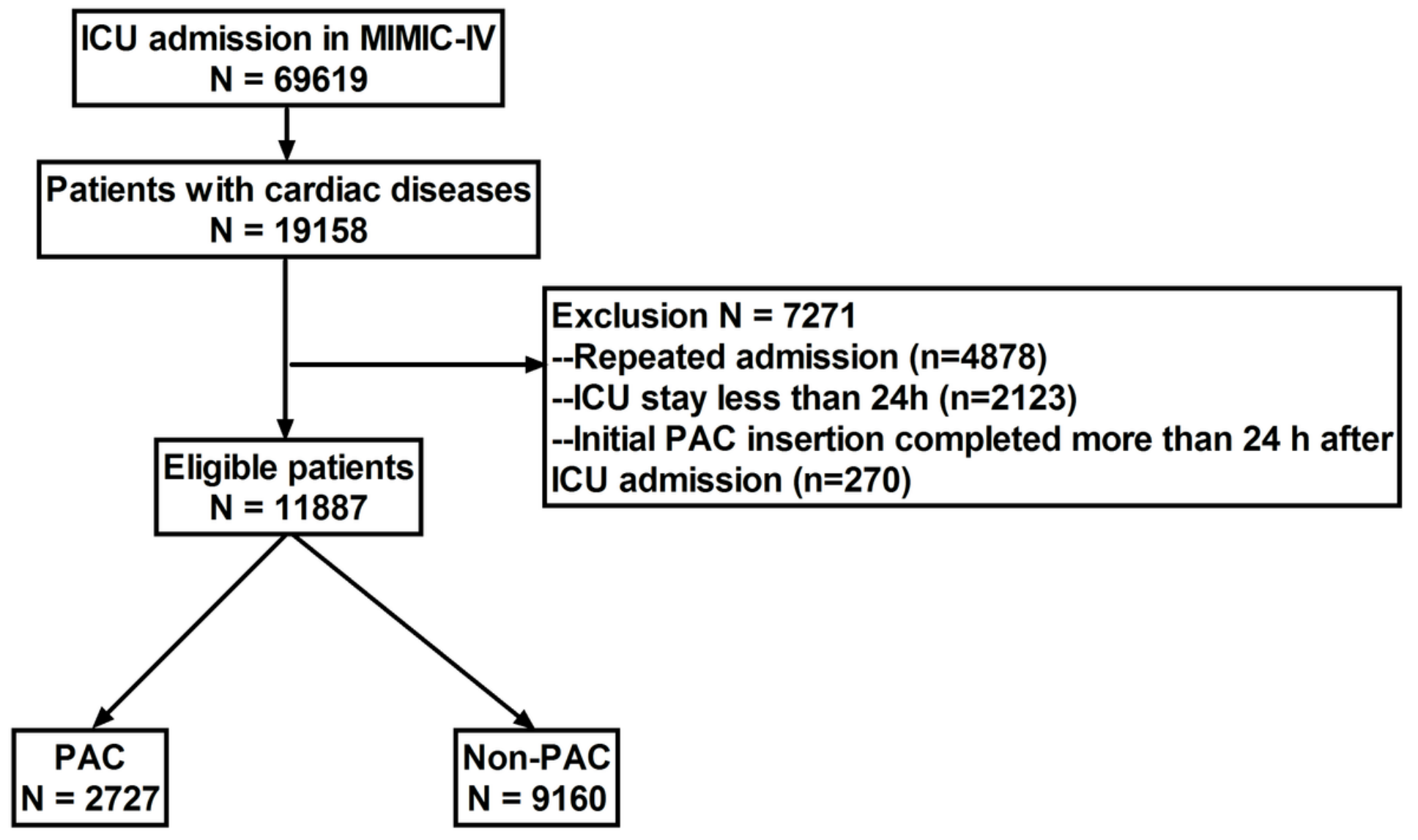

Figure 1

Study cohort 


\section{Outcomes}

\section{Before PSM}

28-day mortality

90-day mortality

Ventilation-free days

Vasopressor-free days

Incidence of AKI

IV Fluid

\section{After PSM}

28-day mortality

90-day mortality

Ventilation-free days

Vasopressor-free days

Incidence of AKI

IV Fluid
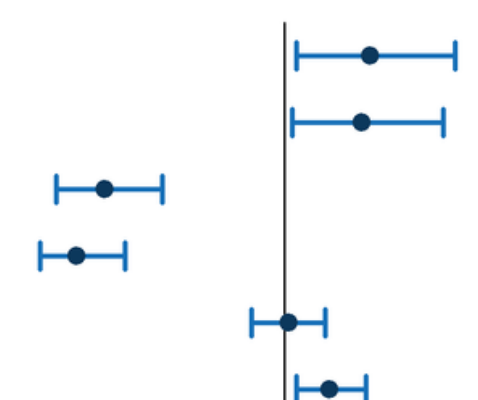

OR $(95 \% \mathrm{Cl}) \quad \mathrm{P}$ value

1.21(1.03-1.42) $\quad 0.02$

1.19(1.02-1.39) $\quad 0.029$

$0.56(0.44-0.70)<0.001$

$0.49(0.40-0.61)<0.001$

1.01(0.92-1.10) $\quad 0.873$

1.11(1.03-1.20) $\quad 0.01$
$0.93(0.70-1.22) \quad 0.578$

$0.90(0.69-1.18) \quad 0.454$

$0.52(0.41-0.65)<0.001$

$0.44(0.35-0.55)<0.001$

1.08(0.93-1.24) 0.306

1.14(1.04-1.25) $\quad 0.007$

Figure 2

The association of PAC and outcomes before and after PSM

\section{Supplementary Files}

This is a list of supplementary files associated with this preprint. Click to download.

- supplementaryinformation.docx 